\title{
Electric dipole moment of the neutron in two-Higgs-doublet models with flavor changing couplings
}

\author{
Jan O. Eeg* \\ Department of Physics, University of Oslo, P.O. Box 1048 Blindern, N-0316 Oslo, Norway
}

(Received 3 June 2020; accepted 20 October 2020; published 12 November 2020)

\begin{abstract}
I consider contributions to the neutron electric dipole moment within two-Higgs-doublet models which allow for small flavor changing neutral Higgs couplings. In a previous paper, I considered flavor changing interactions for the Standard Model Higgs boson to first order in the flavor changing coupling. In that paper I found that the obtained value of the neutron electric dipole moment was below the present experimental limit, given previous restrictions on such couplings. Because this was an effective theory, the result depended on an ultraviolet cut off $\Lambda$, parametrized as $\ln \left(\Lambda^{2}\right)$. In the present paper I demonstrate that, when going to two-Higgs-doublet models, the result stays the same as in the previous paper, up to $M_{\mathrm{SM}}^{2} / M_{H}^{2}$ corrections, where $M_{\mathrm{SM}}$ is the mass of the top quark or the $W$ boson. $M_{H}$ is the mass of the heavy neutral scalar Higgs boson $H$ which is much heavier than the light neutral Higgs boson $h$ with mass $M_{h}$. In the limit $M_{H}^{2} \gg M_{h}^{2}$, the $\ln \left(\Lambda^{2}\right)$ behavior in the previous paper is replaced by $\ln \left(\widetilde{M_{H}}{ }^{2}\right)$, where $\widetilde{M_{H}}$ is of order $M_{H}$. I also explain how some divergences due to exchange of the pseudoscalar Higgs $A$ are canceled by similar contributions from the scalar heavy Higgs $H$, and that these contributions, and finite contributions from $A$ exchange, are suppressed.
\end{abstract}

DOI: 10.1103/PhysRevD.102.095009

\section{INTRODUCTION}

Studies of $C P$-violating phenomena are important in order to understand the asymmetry between matter and antimatter in the Universe. Electric dipole moments (EDMs) of elementary particles violate time reversal symmetry. Assuming $C P T$ symmetry to be valid, EDMs are therefore also $C P$ violating. EDMs of elementary particles have not been seen yet. But experimental searches for EDMs and theoretical studies of EDMs are important because any measured result bigger than the tiny values obtained within the Standard Model (SM) will signal new physics. Reviews on EDMs within the SM and beyond are given in [1-4]. For the EDM of the neutron $\left(n \mathrm{EDM}=d_{n}\right)$ discussed in this paper, the present experimental bound is [5]

$$
d_{n}^{\exp } / e \leq 2.9 \times 10^{-26} \mathrm{~cm}
$$

The corresponding values calculated within the SM are ranging from $10^{-34} e$ to $10^{-31} e \mathrm{~cm}$, depending on the considered mechanism [6-15]. Many models beyond the SM (BSM) give bigger values compared to those obtained

j.o.eeg@fys.uio.no

Published by the American Physical Society under the terms of the Creative Commons Attribution 4.0 International license. Further distribution of this work must maintain attribution to the author(s) and the published article's title, journal citation, and DOI. Funded by SCOAP ${ }^{3}$. within the SM [1-4,16-26]. In the presence of new physics, flavor physics may also give useful $C P$-violating observables. These may occur for instance in $C P$-violating mesonic decays $[17,18,27]$. Within new physics models describing such processes there might be mechanisms that also generate new contributions to the electric dipole moments of quarks (see e.g., [28]).

The electric dipole moment of a single fermion has the form

$$
\mathcal{L}_{f \mathrm{EDM}}=\frac{i}{2} d_{f} \overline{\psi_{f}} \sigma_{\mu \nu} F^{\mu \nu} \gamma_{5} \psi_{f},
$$

where $d_{f}$ is the electric dipole moment of the fermion, $\psi_{f}$ is the fermion (quark) field, $F^{\mu \nu}$ is the electromagnetic field tensor, and $\sigma_{\mu \nu}=i\left[\gamma_{\mu}, \gamma_{\nu}\right] / 2$ is the dipole operator in Dirac space.

We still do not know in detail the properties and couplings of the Higgs boson. For instance, the SM Higgs might mix with a higher mass scalar(s) in the BSM. It has been suggested [29-34] that the SM Higgs boson might have small flavor changing couplings to fermions. Such couplings may also be $C P$ violating. Studying flavor changing processes like $K-\bar{K}, D-\bar{D}$, and $B-\bar{B}$ mixings, and also leptonic flavor changing decays like $\mu \rightarrow e \gamma$ and $\tau \rightarrow \mu \gamma$, bounds on quadratic expressions of such flavor changing couplings were obtained. For the leptonic cases two-loop diagrams of Barr-Zee type [35] for EDMs were also 
considered [29-31,36,37]. Extra couplings of the SM Higgs to quarks has also been considered in [38].

In a previous paper [39], I extended the analysis of EDMs of light quarks with flavor changing SM Higgs couplings $(\mathrm{FCH})$ to two-loop diagrams. Going from one- to two-loop diagrams there is a priori a loop suppression. However, in general, it is known that some two-loop diagrams might give bigger amplitudes than one-loop diagrams because of helicity flip(s) in the latter [30,31,36,40]. And further, what is most important in the present case, I calculated two-loop diagrams containing a small FCH to first order only, in contrast to one-loop contributions with a small FCH coupling to second order, as in [30,31]. Therefore, if the FCH couplings exist, my two-loop amplitudes might numerically compete or even dominate over the one-loop amplitudes calculated previously.

Some of the two-loop diagrams were divergent and parametrized by a ultraviolet cutoff $\Lambda$ [39]. In the present paper I address the same diagrams within two-Higgsdoublet models (2HDMs). In such renormalizable models one knows that the final result does not depend on divergent contributions. In Sec. VI demonstrate how divergent terms disappear due to cancellations of different terms in the general 2HDMs. And I find how the phenomenological FCH coupling of Refs. $[30,31]$ is expressed within 2 HDMs.

For descriptions of $2 \mathrm{HDMs}$, see the review by Branco et al. [41], and also more recent papers [42-53]. Phenomenological consequences of 2HDMs are given in [54].

Some technical details from the two-loop calculations are given in the Appendix.

\section{FLAVOR CHANGING PHYSICAL HIGGS?}

Within the framework in [29-33] the effective interaction Lagrangian for a flavor transition between fermions of the same charge due to SM Higgs boson exchange might be obtained from six-dimensional nonrenormalizable Higgs-type Yukawa-like interactions as shown explicitly in $[31,34]$,

$\mathcal{L}^{(D)}=-\lambda_{i j}\left(\overline{Q_{L}}\right)_{i} \phi\left(d_{R}\right)_{j}-\frac{\tilde{\lambda}_{i j}}{\Lambda_{N P}^{2}}\left(\overline{Q_{L}}\right)_{i} \phi\left(d_{R}\right)_{j}(\phi)^{\dagger} \phi+$ H.c.,

where the generation indices $i$ and $j$ running from 1 to 3 are understood to be summed over; i. e. $d_{j}=d, s, b$ for $j=1$, 2 2, 3. Further, $\phi$ is the SM Higgs $S U(2)_{L}$ doublet field, $\left(Q_{L}\right)_{i}$ are the left-handed $S U(2)_{L}$ quark doublets, and the $\left(d_{R}\right)_{j}$ 's are the right-handed $S U(2)_{L}$ singlet $d$-type quarks in a general basis. Further, $\Lambda_{N P}$ is the scale where new physics is assumed to appear. There is a similar term $\mathcal{L}^{(U)}$ like the one in (3) for right-handed-type $u$-quarks, $u_{j}$, i.e., $u_{j}=u, c, t$ for $j=1,2$, 3. If higher states from a renormalized theory are integrated out. interactions may occur like in (3) below.
In such cases the Yukawa interaction for the SM neutral Higgs boson $h^{0}$ to $d$-type quarks has the form

$$
\mathcal{L}_{Y}^{(D)}=-h^{0}\left(\bar{d}_{L}\right)_{i}\left(Y_{R}^{(D)}\right)_{i j}\left(d_{R}\right)_{j}+\text { H.c. },
$$

where

$$
Y_{R}^{(D)}=\frac{\mathcal{M}^{(D)}}{v}-\epsilon_{R}^{(D)}
$$

Here $\mathcal{M}^{(D)}$ is the mass matrix for $d$-type quarks giving the SM coupling, and $\epsilon_{R}^{(D)}$ is the part which goes beyond the $\mathrm{SM}$, related to the six-dimensional operators in (4). Explicitly, one finds [31,34]

$\left(\mathcal{M}^{(D)}\right)_{i j}=\frac{v}{\sqrt{2}}\left(\lambda_{i j}+\frac{v^{2} \tilde{\lambda}_{i j}}{2 \Lambda_{N P}^{2}}\right), \quad$ and $\quad\left(\epsilon_{R}^{(D)}\right)_{i j}=\frac{v^{2} \tilde{\lambda}_{i j}}{\sqrt{2} \Lambda_{N P}^{2}}$.

As usual $v$ is the vacuum value $246 \mathrm{GeV}$ for the SM Higgs field. The mass matrix $\mathcal{M}^{(D)}$ may be rotated to diagonal form. However, this rotation will in general not give a diagonal $\epsilon_{R}^{(D)}$, such that the SM Higgs coupling to fermions will in general be flavor changing. Thus, for $i \neq j$, $Y_{R}^{(D)}=-\epsilon_{R}^{(D)}$. In [29-34] bounds of FCH couplings to second order are obtained from various flavor changing processes. In my own case, I will need the bound on $Y_{R}^{(D)}(d \rightarrow b)$ from $B_{d}-\overline{B_{d}}$ mixing [31].

\section{YUKAWA INTERACTIONS FOR 2HDM}

For 2HDMs the extended Yukawa interactions for righthanded-type $d$-quarks may then, in the most general case, be written as [46]

$-\mathcal{L}_{\Gamma}^{(D)}=\left(\overline{\left(Q_{L}\right)_{i}^{0}}\right)^{r}\left[\left(\Gamma_{1}\right)_{i j}^{r s}\left(\Phi_{1}\right)^{s}+\left(\Gamma_{2}\right)_{i j}^{r s}\left(\Phi_{2}\right)^{s}\right]\left(d_{R}\right)_{j}^{0}+$ H.c.,

where $i, j$ are as before generation indices running from one to three and $r, s$ are $S U(2)_{L}$ indices running from one to two. The upper index 0 denotes the fields before diagonalization of the mass matrices in the quark sector. Thus the $\Gamma$ 's are $2 \times 2$-dimensional in $S U(2)_{L}$ space and $3 \times 3$-dimensional in generation space. The fields $\Phi_{1,2}$ are the two Higgs fields.

For the right-handed-type $u$-quarks one has similarly as (7)

$$
-\mathcal{L}_{\Delta}^{(U)}={\overline{\left(Q_{L}\right)^{0}}}^{0}\left[\Delta_{1} \tilde{\Phi}_{1}+\Delta_{2} \tilde{\Phi}_{2}\right]\left(u_{R}\right)^{0}+\text { H.c. },
$$

where the generation and $S U(2)_{L}$ indices are suppressed. $\Gamma_{1,2}$ and $\Delta_{1,2}$ are in general complex and independent quantities. In many papers one discusses restrictions on 
2HDMs to avoid flavor changing neutral currents completely. But in this paper the point is to study such potential effects.

The two Higgs doublets may for $n=1,2$ be written $[41,44]$

$$
\begin{aligned}
& \Phi_{n}=e^{i \xi_{n}}\left(\begin{array}{c}
\phi_{n}^{+} \\
\frac{1}{\sqrt{2}}\left(v_{n}+\rho_{n}+i \eta_{n}\right)
\end{array}\right), \\
& \tilde{\Phi}_{n}=e^{-i \xi_{n}}\left(\begin{array}{c}
\frac{1}{\sqrt{2}}\left(v_{n}+\rho_{n}-i \eta_{n}\right) \\
-\left(\phi_{n}^{+}\right)^{\dagger}
\end{array}\right),
\end{aligned}
$$

where $\tilde{\Phi}_{n} \equiv i \sigma_{2} \Phi_{n}^{*}$, and $e^{i \xi_{n}}$ are phase factors. One introduces the parameter $\beta$ through

$$
\tan \beta \equiv \frac{v_{2}}{v_{1}} .
$$

After diagonalization of the mass matrix for the neutral fields $\rho_{1,2}$ obtained from the Higgs potential [41] one finds the neutral scalar mass eigenstates

$$
h=\rho_{1} s_{\alpha}-\rho_{2} c_{\alpha} ; \quad H=-\rho_{1} c_{\alpha}-\rho_{2} s_{\alpha},
$$

and the inverted relations are

$$
-\rho_{1}=-H c_{\alpha}-s_{\alpha} h ; \quad-\rho_{2}=h c_{\alpha}-H s_{\alpha} .
$$

Here $s_{\alpha} \equiv \sin \alpha$ and $c_{\alpha} \equiv \cos \alpha$, where $\alpha$ is the mixing angle coming from the diagonalization of the mass matrix of the $\rho_{1,2}$ fields. Note that in the previous paper [39] the SM Higgs was denoted $H$. In the present paper this symbol is reserved for the heavy neutral Higgs boson within 2HDMs.

In 2HDMs one often uses the Higgs basis, where the doublet fields $H_{1,2}$ are defined by

$e^{-i \xi_{1}} \Phi_{1}=c_{\beta} H_{1}+s_{\beta} H_{2} ; \quad e^{-i \xi_{2}} \Phi_{2}=s_{\beta} H_{1}-c_{\beta} H_{2}$,

where $c_{\beta} \equiv \cos \beta$ and $s_{\beta} \equiv \sin \beta$. With this definition $H_{1}$ has a vacuum value $v=\sqrt{v_{1}^{2}+v_{2}^{2}}$ and $H_{2}$ has zero vacuum value. Thus, in this basis

$H_{1}=\left(\begin{array}{c}G^{+} \\ \frac{1}{\sqrt{2}}\left(v+h^{0}+i G^{0}\right)\end{array}\right), \quad H_{2}=\left(\begin{array}{c}H^{+} \\ \frac{1}{\sqrt{2}}\left(R^{0}+i A\right)\end{array}\right)$,

where $v$ is the vacuum value $246 \mathrm{GeV}$ for the SM Higgs field, and $G^{+}$and $G^{0}$ are Goldstone fields. $H^{+}$is the charged Higgs field and $A$ the neutral pseudoscalar field within 2HDMs. Now the neutral scalar fields $h^{0}$ and $R^{0}$ can be written in terms of the physical (within 2HDMs) neutral scalars $h$ and $H$ as

$$
h^{0}=-c_{\theta} H+s_{\theta} h ; \quad R^{0}=s_{\theta} H+c_{\theta} h,
$$

where

$c_{\theta} \equiv \cos \theta, \quad$ and $\quad s_{\theta} \equiv \sin \theta, \quad$ where $\theta \equiv \alpha-\beta$,

will be the mixing angle in the neutral Higgs sector. Assuming the SM field $h^{0}$ to be close to $h$ means that $\sin \theta$ is close to 1 .

In the Higgs basis the extended Yukawa interactions may (in matrix notation) then be written

$$
\begin{aligned}
-\frac{v}{\sqrt{2}} \mathcal{L}_{Y}= & {\overline{Q_{L}}}^{0}\left(M_{d}^{0} H_{1}+N_{d}^{0} H_{2}\right) d_{R}^{0} \\
& +{\overline{Q_{L}}}^{0}\left(M_{u}^{0} \tilde{H}_{1}+N_{u}^{0} \tilde{H}_{2}\right) u_{R}^{0}+\text { H.c. },
\end{aligned}
$$

where

$$
\begin{aligned}
& M_{d}^{0}=\left(c_{\beta} \Gamma_{1}+e^{i \xi_{\beta}} s_{\beta} \Gamma_{2}\right) \frac{v e^{i \xi_{1}}}{\sqrt{2}}, \\
& N_{d}^{0}=\left(s_{\beta} \Gamma_{1}-e^{i \xi} c_{\beta} \Gamma_{2}\right) \frac{v e^{i \xi_{1}}}{\sqrt{2}},
\end{aligned}
$$

where $\xi=\xi_{2}-\xi_{1}$ and for the $u$-quark case

$$
\begin{aligned}
& M_{u}^{0}=\left(c_{\beta} \Delta_{1}+e^{-i \xi_{\beta}} s_{\beta} \Delta_{2}\right) \frac{v e^{-i \xi_{1}}}{\sqrt{2}}, \\
& N_{u}^{0}=\left(s_{\beta} \Delta_{1}-e^{-i \xi} c_{\beta} \Delta_{2}\right) \frac{v e^{-i \xi_{1}}}{\sqrt{2}} .
\end{aligned}
$$

Now one transforms the mass matrices $M_{d, u}^{0}$ to diagonal form with matrices $U_{R, L}^{d, u}$,

$$
\begin{aligned}
M_{d} & =\left(U_{L}^{d}\right)^{\dagger} M_{d}^{0} U_{R}^{d}=\operatorname{diag}\left(m_{d}, m_{s}, m_{b}\right), \\
N_{d} & =\left(U_{L}^{d}\right)^{\dagger} N_{d}^{0} U_{R}^{d}, \quad d_{R, L}=\left(U_{R, L}^{d}\right)^{\dagger} d_{R, L}^{0},
\end{aligned}
$$

and similarly

$$
\begin{aligned}
& M_{u}=\left(U_{L}^{u}\right)^{\dagger} M_{u}^{0} U_{R}^{u}=\operatorname{diag}\left(m_{u}, m_{c}, m_{t}\right), \\
& N_{u}=\left(U_{L}^{u}\right)^{\dagger} N_{u}^{0} U_{R}^{u}, \quad u_{R, L}=\left(U_{R, L}^{u}\right)^{\dagger} u_{R, L}^{0},
\end{aligned}
$$

for the $u$-quark case.

The total neutral Yukawa interactions for $d$-type quarks may now be written in terms of physical quantities as [46]

$$
\begin{aligned}
-v \mathcal{L}_{Y}^{(d, n)}= & \overline{d_{L}}\left(v+i G^{0}-c_{\theta} H+s_{\theta} h\right) M_{d} d_{R} \\
& +\overline{d_{L}}\left(s_{\theta} H+c_{\theta} h+i A\right) N_{d} d_{R}+\text { H.c. }
\end{aligned}
$$

and for the $u$-quark case the corresponding interactions similarly 


$$
\begin{aligned}
-v \mathcal{L}_{Y}^{(u, n)}= & \overline{u_{L}}\left(v-i G^{0}-c_{\theta} H+s_{\theta} h\right) M_{u} u_{R} \\
& +\overline{u_{L}}\left(s_{\theta} H+c_{\theta} h-i A\right) N_{u} u_{R}+\text { H.c. }
\end{aligned}
$$

For the charged interactions one obtains

$$
\begin{aligned}
-\frac{v}{\sqrt{2}} \mathcal{L}_{Y}^{\text {(charged) }}= & \overline{u_{L}} V_{\mathrm{CKM}}\left(G^{+} M_{d}+H^{+} N_{d}\right) d_{R} \\
& -\overline{d_{L}} V_{\mathrm{CKM}}^{\dagger}\left(G^{-} M_{u}+H^{-} N_{u}\right) u_{R} \\
& + \text { H.c. }
\end{aligned}
$$

While the mass matrices $M_{d}$ and $M_{u}$ are now flavor diagonal, the matrices $N_{d}$ and $N_{u}$ are in general flavor nondiagonal and $C P$ violating. These may give contributions to the $Y_{R}$ 's in Eq. (4). In Eqs. (22) and (23) one observes that there will be flavor changes for the lightest Higgs $h$ proportional to the nondiagonal matrices $N_{d}$ and $N_{u}$.

In many papers on $2 \mathrm{HDMs}$, one assumes for instance an extra discrete symmetry (symmetries) to simplify the theory. In [46] possible restrictions on (7) and (8) are discussed. Here I stick to the general case.

Further, I consider how the six-dimensional interaction in Eq. (3) is obtained in 2HDMs. One way might be to consider the part of the Higgs potential containing a product of four $\Phi_{1}$ or $\Phi_{2}$ Higgs fields (see for example [44]),

$$
\begin{aligned}
V_{2 H D M}^{4 \Phi}= & \frac{1}{2} \lambda_{1}\left(\Phi_{1}^{\dagger} \Phi_{1}\right)^{2}+\frac{1}{2} \lambda_{2}\left(\Phi_{2}^{\dagger} \Phi_{2}\right)^{2}+\lambda_{3}\left(\Phi_{1}^{\dagger} \Phi_{1}\right)\left(\Phi_{2}^{\dagger} \Phi_{2}\right) \\
& +\lambda_{4}\left(\Phi_{1}^{\dagger} \Phi_{2}\right)\left(\Phi_{2}^{\dagger} \Phi_{1}\right)+\frac{1}{2}\left[\lambda_{5}\left(\Phi_{1}^{\dagger} \Phi_{2}\right)^{2}+\text { H.c. }\right] \\
& +\left(\left[\lambda_{6}\left(\Phi_{1}^{\dagger} \Phi_{1}\right)+\lambda_{7}\left(\Phi_{2}^{\dagger} \Phi_{2}\right)\right]\left(\Phi_{1}^{\dagger} \Phi_{2}\right)+\text { H.c. }\right) .
\end{aligned}
$$

Such potentials may contribute to $Y_{R}$ 's in (4). In (3) $\phi$ is the SM Higgs which one within 2HDMs identify with $H_{1}$ in (14). Inserting (13) in (25), the Higgs potential (25) will contain several terms of the type $\left(H_{1}^{\dagger} H_{1}\right)\left(H_{2}^{\dagger} H_{1}\right)$. The coefficient for the sum of such terms is

$$
\begin{aligned}
C_{\lambda}= & \lambda_{1} s_{\beta} c_{\beta}^{3}+\lambda_{2} c_{\beta} s_{\beta}^{3}+\left(\lambda_{3}+\lambda_{4}\right) s_{\beta} c_{\beta}\left(s_{\beta}^{2}-c_{\beta}^{2}\right) \\
& +\lambda_{5} e^{-2 i \xi} s_{\beta}^{3} c_{\beta}-\lambda_{5}^{*} e^{2 i \xi} c_{\beta}^{3} s_{\beta}+2 \lambda_{6} c_{\beta}^{2} s_{\beta}^{2} e^{-i \xi} \\
& +\lambda_{6}^{*}\left(s_{\beta}^{2} c_{\beta}^{2}-c_{\beta}^{4}\right) e^{i \xi}+\lambda_{7}\left(s_{\beta}^{4}-s_{\beta}^{2} c_{\beta}^{2}\right) e^{-i \xi} \\
& -2 \lambda_{7}^{*} c_{\beta}^{2} s_{\beta}^{2} e^{i \xi} .
\end{aligned}
$$

Now the field $H_{2}^{\dagger}$ at space-time $z_{2}$ in such expressions might be contracted with the field $H_{2}$ at space-time $z_{1}$ in (17). This makes the field contraction $(\mathcal{C})$

$$
\mathcal{C}\left(H_{2}\left(z_{1}\right)\left(H_{2}\left(z_{2}\right)\right)^{\dagger}\right)=D_{H_{2}}\left(z_{1}-z_{2}\right) .
$$

Then one obtains an effective six-dimensional interaction

$$
\begin{aligned}
& -\frac{v}{\sqrt{2}} \mathcal{L}_{6}^{(D)}=\left({\overline{\left(Q_{L}\left(z_{1}\right)\right)^{0}}}^{0}\right)\left[M_{d} H_{1}\left(z_{1}\right)\right. \\
& \left.+C_{\lambda} N_{d} D_{H_{2}}\left(z_{1}-z_{2}\right) H_{1}\left(z_{2}\right)\left(\left(H\left(z_{2}\right)_{1}\right)^{\dagger} H_{1}\left(z_{2}\right)\right)\right] \\
& \times\left(d_{R}\right)_{j}^{0}\left(z_{1}\right)+\text { H.c. }
\end{aligned}
$$

So far, this is not a local operator. The propagator $D_{H_{2}}\left(z_{1}-z_{2}\right)$ contains a propagator $D_{h}$ for the light neutral Higgs and a part $D_{H}$ for the heavy neutral Higgs $H$. However, if the SM Higgs is close to the light Higgs $h$, then $s_{\theta}$ is close to 1 , and thereby the scalar $R_{0}$ is close to the heavy Higgs $H$. The part containing the heavy Higgs $H$ interaction is then for $M_{H}^{2} \gg M_{h}^{2}$,

$$
D_{H}\left(z_{1}-z_{2}\right) \simeq-\frac{\delta^{(4)}\left(z_{1}-z_{2}\right)}{M_{H}^{2}}(\sin \theta)^{2},
$$

making the $H$-part of the interaction local in this limit and giving the following nondiagonal contribution to $Y_{R}$ in (4) and (6) for $i \neq j$ :

$$
\begin{aligned}
\left(\left(Y_{R}^{(D)}\right)_{i j}\right)_{H} & =-\left(\left(\epsilon_{R}^{(D)}\right)_{i j}\right)_{H}=\frac{v^{2}\left(\tilde{\lambda}_{i j}\right)_{H}}{\sqrt{2} \Lambda_{N P}^{2}} \\
& \simeq-C_{\lambda}\left(N_{d}\right)_{i j} \frac{v}{\sqrt{2} M_{H}^{2}}(\sin \theta)^{2} .
\end{aligned}
$$

The nonlocal $h$-part $D_{h}$ would be a term corresponding to a higher order diagram. This term is shortly discussed at the end of Sec. V.

\section{IV. $n$ EDM GENERATED FROM A FLAVOR CHANGING HIGGS COUPLING}

In this section I give a short summary of the results from the previous paper [39]. The reason being that the diagrams calculated in that paper are also relevant in 2HDMs.

In [39], two classes of diagrams for EDMs of light quarks, shown in Figs. 1 and 2, were considered. These diagrams are obtained from the flavor nondiagonal interaction in (4), completed by SM interactions. But these diagrams also give contributions within 2HDMs with flavor change, as explained in the next section.

As explained in [39], the $u$-quark dipole moment $d_{u}$ due to diagrams in Fig. 1 are suppressed and therefore neglected. Thus, the $d$-quark dipole moment contributions dominate, and other contributions are neglected. Summing all contributions from diagrams in Figs. 1 and 2, I obtained the dominating contribution in the bare case (before QCD corrections) [39]

$$
\left(\frac{d_{d}}{e}\right)_{\mathrm{Tot}}=F_{2} C_{E}\left(\mu_{\Lambda}\right) \operatorname{Im}\left[Y_{R}(d \rightarrow b) V_{t d}^{*} V_{t b}\right],
$$

where the constant $F_{2}$ sets the overall scale of the EDMs obtained from the two-loop diagrams 

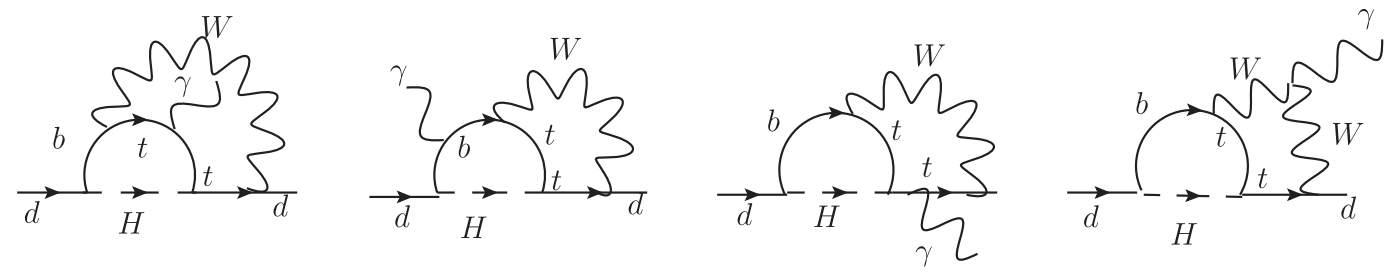

FIG. 1. The first class of diagrams contain the FCH coupling and the big Higgs-top coupling proportional to the top mass $m_{t}$. For the first three diagrams, there are also corresponding diagrams where the $W$ boson is replaced by an Goldstone Higgs boson within Feynman gauge. In this figure $H$ may denote the SM Higgs $h^{0}$. Further, within the 2HDMs $H$ may denote the lightest neutral Higgs boson $h$, the heavier neutral Higgs boson $H$, or the neutral pseudoscalar Higgs $A$.
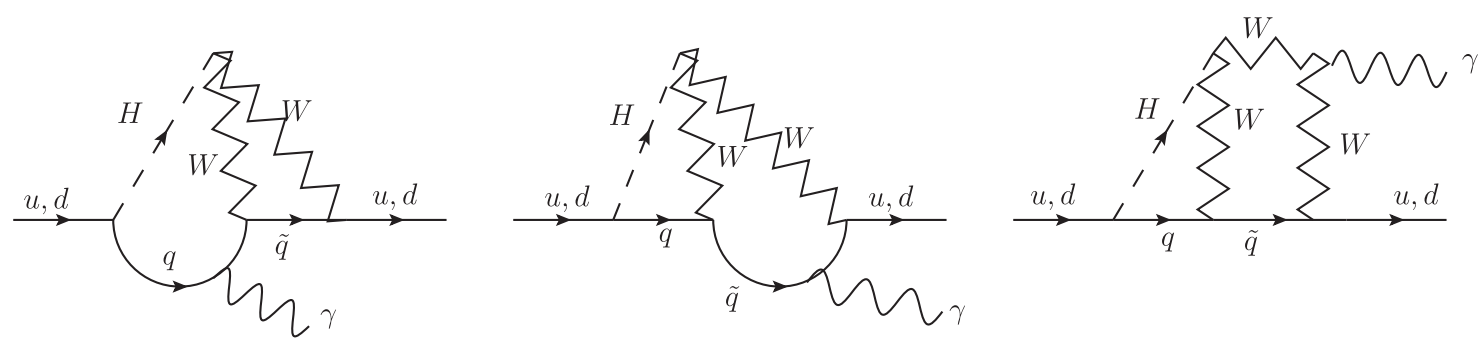

FIG. 2. A class of diagrams containing the FCH coupling and the big $H W W$ coupling proportional to $M_{W}$. Additional graphs with the $W$ replaced by an unphysical (Goldstone) Higgs within Feynman gauge has to be added. In this figure $H$ may denote the SM Higgs $h^{0}$, or within the $2 \mathrm{HDM}$ s the lightest neutral Higgs $h$, the heavier neutral Higgs $H$, or the neutral pseudoscalar Higgs $A$.

$$
\begin{aligned}
F_{2} & =\frac{g_{W}^{3}}{M_{W} \sqrt{2}}\left(\frac{1}{16 \pi^{2}}\right)^{2}=\frac{2 M_{W}^{2}}{v^{3}}\left(\frac{1}{16 \pi^{2}}\right)^{2} \\
& \simeq 6.94 \times 10^{-22} \mathrm{~cm},
\end{aligned}
$$

where $v=246 \mathrm{GeV}$ is the electroweak symmetry breaking scale and where I have used the conversion relation $1 /(200 \mathrm{MeV})=10^{-13} \mathrm{~cm}$.

Further,

$C_{E}\left(\mu_{\Lambda}\right)=\left(\left[\frac{2 u_{t}}{3} p_{1}\left(u_{t}\right)+\frac{25}{12} p_{2}\left(u_{t}\right)\right] C_{\Lambda}+f_{\text {Fin }}\right)$.

Here $p_{1}$ and $p_{2}$ are one-loop functions for finite subloops [39]

$$
\begin{aligned}
& p_{1}(u) \equiv \frac{u}{(u-1)}\left(1-\frac{\ln (u)}{u-1}\right) ; \quad \text { and } \\
& p_{2}(u) \equiv \frac{u}{(u-1)}\left(\frac{u \cdot \ln (u)}{u-1}-1\right) .
\end{aligned}
$$

Using standard values for the masses of $W$ and $t$, one finds numerically

$$
u_{t} \equiv\left(\frac{m_{t}}{M_{W}}\right)^{2} \simeq 4.65 ; \quad p_{1}\left(u_{t}\right)=0,737, \quad p_{2}\left(u_{t}\right)=1.219 .
$$

The UV divergence is parametrized through the quantity

$$
C_{\Lambda} \equiv \ln \left(\frac{\Lambda^{2}}{M_{W}^{2}}\right)+\frac{1}{2},
$$

where $\Lambda$ is the UV cutoff. Numerically, $C_{\Lambda}$ is $\sim 5.5-9.4$ for $\Lambda \sim 1-7 \mathrm{TeV}$. The quantity $f_{\text {Fin }} \simeq-7.7$ is the sum of the diagrams not containing divergent parts and also the finite parts of diagrams containing a divergence. The divergence appears in the $d \rightarrow t W$ subloop in some of the diagrams.

The $V$ 's are Cabibbo-Kobayashi-Maskawa (CKM) matrix elements in the standard notation. We note that because $V_{t d}^{*} V_{t b}$ is complex, there will be an EDM even if $Y_{R}(d \rightarrow b)$ is real.

Using the absolute value of $V_{t d}^{*} V_{t b}$ from [55], one may write my result for the $n \mathrm{EDM}$ in the following way, as shown in [39]

$$
\begin{aligned}
d_{n} / e \simeq & N(\Lambda) \\
& \times\left\{\frac{\left|Y_{R}(b \rightarrow d)\right|}{\left|Y_{R}(b \rightarrow d)\right|_{\text {Bound }}} \cdot \operatorname{Im}\left[\frac{Y_{R}(d \rightarrow b)}{\left|Y_{R}(b \rightarrow d)\right|} \cdot \frac{V_{t d}^{*} V_{t b}}{\left|V_{t d}^{*} V_{t b}\right|}\right]\right\} \\
& \times 10^{-26} \mathrm{~cm},
\end{aligned}
$$

where I have scaled the result with the bound in [30,31] obtained from $B_{d}-\overline{B_{d}}$ mixing,

$$
\left|Y_{R}(d \rightarrow b)\right| \leq 1.5 \times 10^{-4} \equiv\left|Y_{R}(d \rightarrow b)\right|_{\text {Bound }} .
$$

The function $N(\Lambda)$ is defined by the relation 


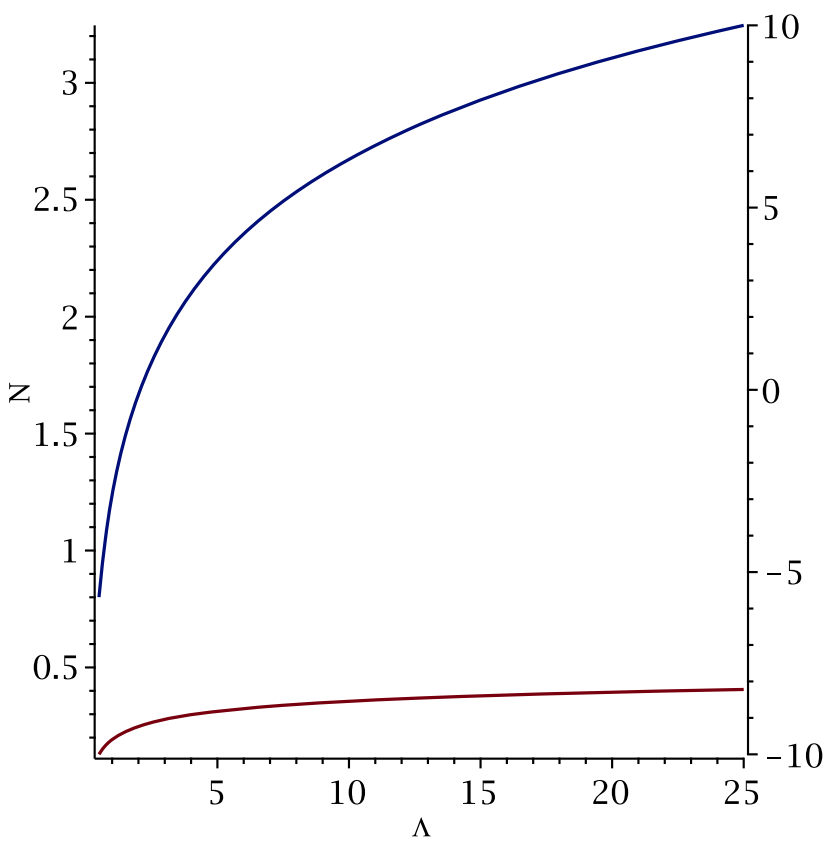

FIG. 3. The quantity $N=N(\Lambda)$, in units $10^{-26} \mathrm{~cm}$, as a function of cutoff $\Lambda$ (in TeV). The blue (upper) curve is for the bare case and the red (lower) curve is for the case when the suppressing QCD corrections are included.

$\rho_{d} F_{2}\left|Y_{R}(d \rightarrow b)\right|_{\text {Bound }}\left|V_{t d}^{*} V_{t b}\right| C_{E}\left(\mu_{\Lambda}\right)=N(\Lambda) \times 10^{-26} \mathrm{~cm}$,

where $\rho_{d} \simeq 0.74$ is the contribution from the $d$-quark $\mathrm{EDM} d_{d}$ to the neutron $\mathrm{EDM} d_{n}$ within lattice calculations 58-56]].

$N(\Lambda)$ is plotted as a function of $\Lambda$ in Fig. 3 for the bare case (at the renormalization scale $\mu=\mu_{\Lambda}$, blue curve) and with QCD corrections (at the hadronic scale $\mu=\mu_{h} \simeq 1 \mathrm{GeV}$, red curve, as explained in [39]).

Now, the maximal value of the parenthesis $\{\ldots\}$ in (37) is $=1$. Thus, if the bound for $Y_{R}(d \rightarrow b)$ in (38) is saturated, the plot for the function $N_{\Lambda}$ in Fig. 3 shows that when the cutoff $\Lambda$ is stretched up to $20 \mathrm{TeV}$, the bound for $n \mathrm{EDM}$ in (1) is reached in the bare case, while the perturbative QCD suppression tells [39] that the value of the $n \mathrm{EDM}$ can at maximum be at most $0.4 \times 10^{-26} e \mathrm{~cm}$ for $\Lambda$ up to $20 \mathrm{TeV}$. If the bound for $\left|Y_{R}(d \rightarrow b)\right|$ is reduced, and also $\Lambda$ is reduced, my value for $n \mathrm{EDM}$ will be accordingly smaller.

\section{THE $n$ EDM IN THE 2HDMs}

All diagrams in Figs. 1 and 2 will also contribute within 2HDMs without restrictions as given in (22) and (23), and one obtains diagrams both with light $h$ and heavy $H$ and $A$ exchanges. Exchange of the pseudoscalar $A$ [as defined in Eq. (14)] does not contribute to the order I work, because it does not couple to the mass matrices $M_{u}$ and $M_{d}$ in (22) and (23). As also is seen from these equations, $A$ couples only to the matrices $N_{u, d}$. Exchanges of $A$ will be treated explicitly later in this section and are shown to be suppressed. For contributions with exchange of $h$ and $H$, the amplitudes are equal, but have opposite signs according to the Eqs. (22) and (23). Explicitly, for $X=h$ and $X=H$ exchanges I find the effective contribution

$$
\left[Y_{R}(d \rightarrow b)\right]_{N}^{\mathrm{eff}}=\frac{1}{v}\left(N_{d}\right)_{b d} \cos \theta \sin \theta,
$$

where $\theta$ is defined in (16) and $\left(N_{d}\right)_{b d}$ in (18). Within 2HDMs, given by (22) and (23), Eq. (40) may the be inserted in (37). Combining (40) and (38) gives then a restriction on flavor changing $2 \mathrm{HDMs}$ from $B_{d}-\overline{B_{d}}$ mixing [31].

As shown in [39] some diagrams with exchange of only one neutral Higgs boson have divergent parts. In the following I will for illustrative purposes consider in some detail the case where a soft photon is emitted from a $W$ boson, as shown in the right panel of Fig. 1. Then the result for this diagram is proportional to the two-loop integral tensor (neglecting $m_{b}$ compared to $m_{t}$ )

$$
T_{\mu \nu}^{W}(X)=\iint \frac{đ p d r K_{\mu} p_{\nu}}{\left(r^{2}-M_{W}^{2}\right)^{2}\left(r^{2}-m_{t}^{2}\right)\left((r+p)^{2}-m_{t}^{2}\right)\left(p^{2}-m_{b}^{2}\right)\left(p^{2}-M_{X}^{2}\right)},
$$

where $M_{X}$ is the mass of either the light or heavy Higgs, i.e., $X=h, H$, later also $A$. Moreover, $K_{\mu}=K_{\mu}^{R}=r_{\mu}$ when a Higgs is coupling to the top quark with a right-handed coupling, and $K_{\mu}=K_{\mu}^{L}=(r+p)_{\mu}$ when this coupling is left-handed. In the latter case, the integral over $₫ p$ diverges. When $X=h$ and $X=H$ couples to the diagonal mass matrix part $\left(M_{u}\right)_{t t}=m_{t}$ one has $K_{\mu} \rightarrow\left(K_{\mu}^{R}+K_{\mu}^{L}\right)=$ $(2 r+p)_{\mu}$.

In the limit where $\sin \theta$ is close to 1 , the $h$-part can be written as in [39]
$T_{\mu \nu}^{W}(h)=\frac{g_{\mu \nu}}{4 m_{t}^{2}}\left(\frac{1}{16 \pi^{2}}\right)^{2}\left(C_{\Lambda} \cdot p_{2}\left(u_{t}\right)+t_{W \mathrm{Fin}}^{L}+t_{W \mathrm{Fin}}^{N}\right)$,

where $C_{\Lambda}$ is given by (36) and where $p_{2}(u)$ is defined in (34) and $u_{t}$ is the mass ratio in (35). Furthermore,

$$
t_{W \mathrm{Fin}}^{L} \simeq-2.8, \quad \text { and } \quad t_{W \mathrm{Fin}}^{N} \simeq-1.1 .
$$

Here $t_{W \text { Fin }}^{L}$ is the finite term following the logarithmic divergence, and $t_{W \text { Fin }}^{N}$ is a completely finite term, as 
explained in the Appendix. For other diagrams there are similar expressions as (43), but with other numbers. Completely finite diagrams have only a term similar to $t_{W F i n}^{N}$, for instance the two first (from left) diagrams in Fig. 1.

One should remember that the loop amplitude in (42) is multiplied by a factor $g_{W} m_{t} / M_{W}$ from the SM Higgs coupling to the top quark. There will also be an extra factor $m_{t}$ from the mass part of one of the top quark propagators. Thus the factor $1 / m_{t}^{2}$ at the right-hand side of (42) will cancel and the quantity $C_{E}$ in (33) is dimensionless. The factor $1 / M_{W}$ from the Higgs to top quark coupling goes into $F_{2}$ in (32).

The individual loop integrals for $X=h$ or $X=H$ alone have divergent parts. But within 2HDMs, one observes from Eqs. (15), (22), and (23) that the terms with exchange of $h$ and $H$ will have opposite signs due to the Cabibbo-like mixing of $h$ and $H$, and one obtains a cancellation of divergences due to exchanges of these two bosons. Thus I use

$$
\frac{1}{\left(p^{2}-M_{h}^{2}\right)}-\frac{1}{\left(p^{2}-M_{H}\right)}=\frac{\left(M_{h}^{2}-M_{H}^{2}\right)}{\left(p^{2}-M_{h}^{2}\right)\left(p^{2}-M_{H}^{2}\right)}
$$

and obtain the total tensor for exchanges of both $h$ and $H$,

$$
\Delta T_{\mu \nu}^{W}=T_{\mu \nu}^{W}(h)-T_{\mu \nu}^{W}(H)=\left(M_{h}^{2}-M_{H}^{2}\right) S_{\mu \nu}^{W},
$$

where

$$
S_{\mu \nu}^{W}=\iint \frac{đ p d r(2 r+p)_{\mu} p_{\nu}}{\left(r^{2}-M_{W}^{2}\right)^{2}\left(r^{2}-m_{t}^{2}\right)\left((r+p)^{2}-m_{t}^{2}\right)\left(p^{2}-m_{b}^{2}\right)\left(p^{2}-M_{h}^{2}\right)\left(p^{2}-M_{H}^{2}\right)},
$$

is finite.

As shown in the Appendix, this loop integral contains logarithmic and dilogarithmic functions of masses of the top quark, the $W$ boson, and the neutral Higgs bosons $h, H$. In the limit $M_{H}^{2} \gg M_{h}^{2}$, I find that

$$
S_{\mu \nu}^{W} \sim \frac{\ln \left(M_{H}^{2}\right)}{M_{\mathrm{SM}}^{2} M_{H}^{2}},
$$

where $M_{\mathrm{SM}}$ is either $m_{t}, M_{h}$, and/or $M_{W}$. I have found the leading result replacing Eq. (31) can be written

$$
\begin{aligned}
\Delta T_{\mu \nu}^{W}= & \left.\frac{g_{\mu \nu}}{4 m_{t}^{2}}\left(\frac{1}{16 \pi^{2}}\right)^{2}\left(\widetilde{\left(C_{H}\right.}\right)^{W} \cdot p_{2}\left(u_{t}\right)+t_{W \mathrm{Fin}}^{L}+t_{W \mathrm{Fin}}^{N}\right) \\
& +\mathcal{O}\left(M_{\mathrm{SM}}^{2} / M_{H}^{2}\right)
\end{aligned}
$$

where corrections are of order $\left(M_{\mathrm{SM}} / M_{H}\right)^{2}$. Here one might expect that the divergent term $C_{\Lambda}$ from (42) is replaced by a finite term where the cutoff $\Lambda$ is replaced by just the heavy neutral Higgs mass $M_{H}$. This is true to leading order, but it turns out that the $\left(\widetilde{C_{H}}\right)^{W}$ is a bit more complicated, as shown in the Appendix,

$$
\left(\widetilde{C_{H}}\right)^{W}=\ln \left[\left(\frac{\left(\widetilde{M_{H}}\right)_{W}}{M_{W}}\right)^{2}\right]+\frac{1}{2},
$$

(up to corrections of order $\left(M_{\mathrm{SM}} / M_{H}\right)^{2}$ as mentioned above) where

$$
\left(\widetilde{M_{H}}\right)_{W}=M_{H} e^{\alpha_{W}}, \quad \alpha_{W} \equiv \frac{\left(\ln u_{t}\right)^{2}}{4\left(1-1 / u_{t}-\left(\ln u_{t}\right)\right)}, \quad e^{\alpha_{W}} \simeq 0.45,
$$

and where $u_{t}$ is given in (35). The term $t_{W \text { Fin }}^{L}$ in (48) is, up to $\left(M_{\mathrm{SM}} / M_{H}\right)^{2}$, the same $t_{W \text { Fin }}^{L}$ as in (42).

It is easy to see that the $t_{W \text { Fin }}^{L}$ 's are the same if one uses the mathematical trick given in (A21) in the Appendix. The term $t_{W \text { Fin }}^{N}$ is trivially the same [up to corrections of order $\left.\left(M_{\mathrm{SM}} / M_{H}\right)^{2}\right]$. For other diagrams, where the soft photon is emitted by a quark $q=b, t$, say, the factor $e^{\alpha_{W}}$ will be replaced by a similar factor $e^{\alpha_{q}}$ of the same order of magnitude. Now the result given by (40) and (48)-(50) can be completed with similar expressions for the rest of diagrams in Figs. 1 and 2. Then the final result will be as in [39], i.e., as in Eq. (37) and Fig. 3, with the cutoff $\Lambda$ replaced by a mass $\widehat{M_{H}}$ of order $M_{H}$ [depending on the various $\alpha$ 's similar to $\alpha_{W}$ in (50)].

Up to now I have considered contributions where $X=h$ and $X=H$ are coupled to the diagonal mass matrix $M_{u} \rightarrow m_{t}$. Now I will consider the contributions where the neutral Higgses $h, H, A$ couple to the diagonal $t t$ element of $N_{u}$. Due to the mixing of the scalars $h$ and $H$ the exchanges of these are given, as seen from (22) and (23), by the propagator terms

$$
\begin{aligned}
& \frac{(\sin \theta)^{2}}{\left(p^{2}-M_{H}^{2}\right)}-\frac{(\cos \theta)^{2}}{\left(p^{2}-M_{h}\right)} \\
& \quad=\frac{1}{\left(p^{2}-M_{H}^{2}\right)}-\frac{(\cos \theta)^{2}\left(M_{H}^{2}-M_{h}^{2}\right)}{\left(p^{2}-M_{h}^{2}\right)\left(p^{2}-M_{H}^{2}\right)} .
\end{aligned}
$$

The last term on the right-hand side will give finite and very small terms because $\cos \theta$ is small. Such terms are then neglected. The first term on the right-hand side will a priori give a divergent term if $H$ has a left-handed coupling to $\left(N_{u}\right)_{t t}$, [i.e., $K_{\mu}=K_{\mu}^{L}=(r+p)_{\mu}$ and $X=H$ in (41)]. However, because of the imaginary coupling of $A$, the 
similar exchange of $A$ will also be divergent and cancels the divergent term from $H$. The cancellation is exact in the limit $M_{A} \rightarrow M_{H}$, and in general there is a finite leftover. It is important to note that such (partial) cancellations occur also before eventual explicit restrictions (symmetry requirements) are assumed for the 2HDMs. When $H$ and $A$ couples right-handed with $\left(N_{u}\right)_{t t}$, they have the same sign and are finite, and they are equal in the limit $M_{A} \rightarrow M_{H}$. In this case one finds a tensor with the leading behavior

$$
T_{\mu \nu}^{W R} \simeq-\left(\frac{1}{16 \pi^{2}}\right)^{2} \frac{g_{\mu \nu}}{32 M^{2}} \ln \left(\frac{M^{2}}{M_{\mathrm{SM}}^{2}}\right)
$$

where $M=M_{H}$ or $M=M_{A}$, and $M_{\mathrm{SM}}$ is of order $M_{W}$ or $m_{t}$. Thus, the contributions from $\left(N_{u}\right)_{t t}$ are suppressed by $\left(M_{\mathrm{SM}} / M\right)^{2}$ compared to the terms with coupling to the mass matrix $M_{u}$ as stated in the beginning of this section. Then, my result has, before QCD corrections, the general structure

$$
\begin{aligned}
\left(\frac{d_{n}}{e}\right) \sim & \left(\frac{1}{16 \pi^{2}}\right)^{2} \frac{g_{W}^{3}}{M_{\mathrm{SM}}}\left[\ln \left(\frac{M_{H}^{2}}{M_{\mathrm{SM}}^{2}}\right)+N L\right] \\
& \cdot \operatorname{Im}\left[Y_{R}(d \rightarrow b) V_{t d}^{*} V_{t b}\right]
\end{aligned}
$$

where $N L$ is a dimensionless nonlogarithmic, nonleading term, depending on $m_{t}, M_{W}$, and the light Higgs mass $M_{h}$. $N L$ also contains the $\left(M_{\mathrm{SM}} / M\right)^{2}$ corrections.

Concerning the six-dimensional interaction in (3), it will for the local part also be proportional to $N_{d}$ as shown in (30) and also suppressed by $\left(v / M_{H}\right)^{2}$. The nonlocal part given by exchange of the light $h$ boson will be of one order higher. The corresponding loop diagram is proportional to

$$
T_{\mu \nu}^{W 6}=S_{\mu \nu}^{W}\left(M_{H}=M_{h}\right)=g_{\mu \nu} \frac{1}{\left(16 \pi^{2}\right)^{2}} \frac{1}{4 M_{W}^{4}} \times 0.015,
$$

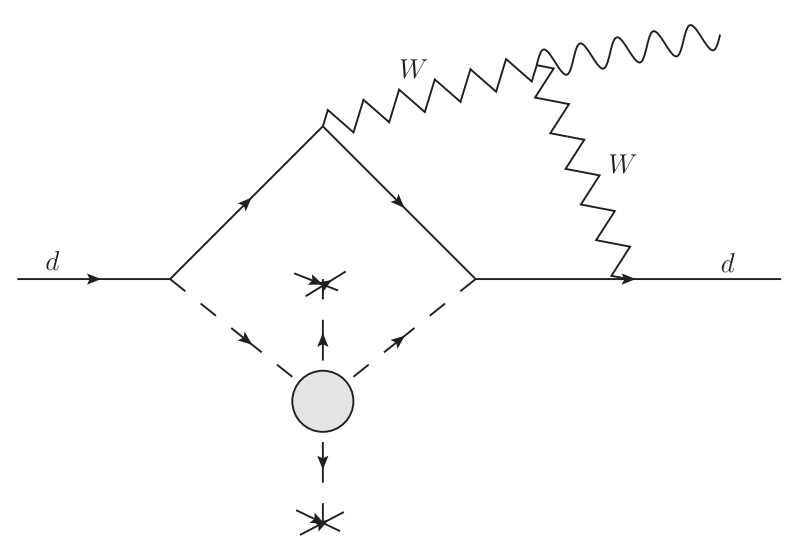

FIG. 4. Diagram generated in the 2HDMs. The gray circle denotes the interaction in (25). The dashed lines are Higgses and the crosses in the end of two of these denotes the Higgs vacuum expectation value. where standard numerical values for masses for SM particles have been used. The contribution from this diagram should also be multiplied by $(\cos \theta)^{2} v^{2}$, and $C_{\lambda}$ in (26), and will be small (because $\cos \theta$ is small when $h$ is close to $h^{0}$ ).

\section{DISCUSSIONS AND CONCLUSIONS}

In previous papers $[30,31]$ based on the effective theory for FCH couplings, one-loop diagrams for the neutron EDM were considered. There is a one-loop diagram for an EDM of the $u$-quark with Higgs and the $t$-quark in the loop which is proportional to the $t$-quark mass and the product of the FCH couplings $Y_{R}(u \rightarrow t)$ and $Y_{R}(t \rightarrow u)$. The absolute values of these FCH couplings are not very restricted, according to [31]

$$
\sqrt{\left|Y_{R}(u \rightarrow t)\right|^{2}+\left|Y_{R}(t \rightarrow u)\right|^{2}} \leq 0.3 .
$$

However, from the $n \mathrm{EDM}$ based on this one-loop diagram one obtains the bound

$$
\operatorname{Im}\left[Y_{R}(u \rightarrow t) \times Y_{R}(t \rightarrow u)\right] \leq 4.3 \times 10^{-7} .
$$

There is a one-loop diagram for an EDM of the $d$-quark with Higgs and the $b$-quark in the loop proportional to the $b$-quark mass and the product of $\mathrm{FCH}$ couplings $Y_{R}(d \rightarrow b)$ and $Y_{R}(b \rightarrow d)$. Bound on the coupling $Y_{R}(d \rightarrow b)$ is given in (38), and one has also [31]

$$
\operatorname{Im}\left[Y_{R}(d \rightarrow b) \times Y_{R}(b \rightarrow d)\right] \leq 6.4 \times 10^{-8} .
$$

Then the $d$-quark EDM with a $b$-quark one-loop diagram could at most give a contribution to the $n \mathrm{EDM}$ of about $1.3 \times 10^{-26} e \mathrm{~cm}$ before QCD corrections are taken into account. But QCD corrections will suppress this result further by a factor of order $10^{-1}$ (see [39] and references therein).

In my previous paper [39] I presented calculations of two-loop diagrams which depended on a flavor changing coupling. Such two-loop contributions are suppressed with the flavor changing coupling to first order only, instead of the second order suppression for one-loop diagrams. A price for going to two loops is in this case a suppression factor $g_{W}^{2} /\left(16 \pi^{2}\right) \simeq 1.2 \times 10^{-3}$ which is numerically bigger than $Y_{R}(d \rightarrow b)$, as seen in Eq. (38). Therefore the twoloop diagrams of Ref. [39] are expected to (more than) compete numerically with the corresponding one-loop diagrams. Also it is important to note that, in the two-loop case considered here, there will be a EDM different from zero even if $Y_{R}(d \rightarrow b)$ is real, because it is combined with a CKM factor.

Going from the effective theory of [30,31,39] to the general 2HDMs in this paper, I have shown that the result from [39] stays the same, up to corrections of order $\left(M_{\mathrm{SM}} / M_{H, A}\right)^{2}$, when the flavor changing coupling [30,31,39] is replaced 
by the corresponding expression in 2HDM, as shown in Eq. (40). This equation then gives a bound for the expression $\left(N_{d}\right)_{b d} \cdot \cos \theta \cdot \sin \theta / v$ within 2HDMs with flavor change. Further, I have shown that the divergences appearing for some EDM diagrams with flavor changing Higgs in my previous paper [39] are, as expected, removed when extending the analysis to two-Higgs-doublet models allowing for flavor changes by neutral scalars. Namely, the divergences $\sim \ln (\Lambda)$ in [39] are replaced by $\ln \left(\widetilde{M_{H}}\right)$, where $\widetilde{M_{H}}$ is equal to $M_{H}$ times a function of the ratio $m_{t} / M_{W}$. [see Eq. (50)]. Finally, I have demonstrated that exchanges of the pseudoscalar Higgs $A$ does not contribute to the order I work because they are suppressed by $\left(M_{\mathrm{SM}} / M_{A}\right)^{2}$. I have also demonstrated that some potential divergences involving exchanges of $A$ are canceled by similar divergences from exchanges of the heavy scalar $H$.

It is also found that the six-dimensional interaction in (3) used in [39] will also be proportional to the nondiagonal $N_{d}$ matrix in (17), (20), and (22). The quantity $C_{\lambda}$ from the Higgs potential contains many unknowns. But this term is suppressed by $\left(v / M_{H}\right)^{2}$, as seen from Eq. (30).

There are also other calculations of the $n \mathrm{EDM}[4,54]$. In [54] EDM for flavor changing couplings are considered, but only at one loop. The results, given by the matrices in Eq. (75) of that paper, are in reasonable agreement with [31], cf. also the Eqs. (55)-(57).

In [4] a 2HDM is considered at two-loop level in terms of Barr-Zee diagrams, but with no flavor change from neutral Higgses. This contribution is suppressed by the mass ratio $m_{f} / M_{W}$, where $m_{f}$ is a light quark mass $m_{u}$ or $m_{d}$. Then, if the nondiagonal elements of the matrix $N_{d}$ are of the same order as the diagonal ones, the result presented in this paper is bigger than in Eq. (53) of [4]. On the other hand, if the nondiagonal element $\left(N_{d}\right)_{b d}$ is very small, the result of [4] might be bigger. And of course, if the nondiagonal elements of $N_{d}$ are restricted to be zero to avoid flavor changing neutral currents completely, then my result is zero.

In general, the mechanism given by the diagrams in Fig. 1 will also work in some other theories with exchanges of scalars and the $W$ boson, for example for an EDM of the electron within leptoquark models [59].

To conclude, when going from [39] to the present study of $2 \mathrm{HDMs}$ with flavor change I have shown the following:

(i) The result from [39] stays unchanged up to corrections of $\left(M_{\mathrm{SM}} / M_{H, A}\right)^{2}$. The logarithmic divergence $\sim \ln \left(\Lambda / M_{W}\right)$ in [39] is replaced by $\ln \left(\widetilde{M_{H}} / M_{W}\right)$, where $\widetilde{M_{H}}$ is of order $M_{H}$.

(ii) The flavor changing coupling $Y_{R}(d \rightarrow b)$ in $[30,31,39]$ is found to be replaced by $\left(N_{d}\right)_{b d} \cdot \cos \theta$. $\sin \theta / v$ in a $2 \mathrm{HDMs}$ with flavor changing neutral Higgses. I have also identified an example of the six- dimensional term in Eq. (3) which was a starting point in $[31,34,39]$.

(iii) There is a cancellation between divergent terms with $A$ and $H$ exchanges.

(iv) There is a suppression $\left(M_{\mathrm{SM}} / M_{H, A}\right)^{2}$ of finite terms due to exchange of $A$ and $H$ terms not coupled to the top mass.

\section{ACKNOWLEDGMENTS}

I thank Svjetlana Fajfer for useful comments.

\section{APPENDIX: DETAILS FOR THE LOOP INTEGRALS}

If the soft photon is emitted from the $W$ boson as in the left diagram in Fig. 1, then the left subloop containing the Higgs boson is logarithmically divergent. The result of the divergent part of (41) can be written

$$
\begin{aligned}
T_{\mu \nu}(h)= & \frac{g_{\mu \nu}}{4} 2 ! \int_{0}^{1} d x \int_{0}^{(1-x)} d y \\
& \times \int \frac{d r}{\left(r^{2}-M_{W}^{2}\right)^{2}\left(r^{2}-m_{t}^{2}\right)}\left(I_{2}(R)+R \cdot I_{3}(R)\right),
\end{aligned}
$$

where the quantity $R$ depends on the squared loop momentum $r^{2}$. For $n=2,3$

$$
I_{n}(R)=\int \frac{d p}{\left(p^{2}-R\right)^{n}} .
$$

Then for cutoff regularization,

$$
\left(I_{2}(R)+R \cdot I_{3}(R)\right)=\frac{i}{16 \pi^{2}}\left(\ln \left(\Lambda^{2} / R\right)-\frac{3}{2}\right),
$$

where $\Lambda$ is the cutoff, and $x$ and $y$ are Feynman parameters, and

$$
\begin{aligned}
& R \equiv B-x(1-x) r^{2} \\
& B \equiv m_{b}^{2}+x\left(M_{W}^{2}-m_{b}^{2}\right)+y\left(M_{h}^{2}-m_{b}^{2}\right) .
\end{aligned}
$$

One may split up

$$
\begin{aligned}
\left(\ln \left(\Lambda^{2} / R\right)-\frac{3}{2}\right)= & \left(\ln \left(\Lambda^{2} / M_{W}^{2}\right)-\frac{3}{2}\right) \\
& +\ln \left(M_{W}^{2} / R\right)
\end{aligned}
$$

where the first term corresponds to $C_{\Lambda}$ in (36), and the $\ln \left(M_{W}^{2} / R\right)$ term corresponds to $t_{W \text { Fin }}^{L}$. There is also a finite term $t_{W \text { Fin }}^{N}$ corresponding to an completely finite extra term $\sim 1 / R$ not shown in (A1). We also note that the one-loop integral 


$$
K_{W}=\int \frac{d r}{\left(r^{2}-M_{W}^{2}\right)^{2}\left(r^{2}-m_{t}^{2}\right)}=\frac{-i}{16 \pi^{2} m_{t}^{2}} p_{2}\left(u_{t}\right),
$$

where $p_{2}(u)$ defined in (34) is the proportionality factor for the divergent term $C_{\Lambda}$ in (36).

Now I consider the finite loop integral in (46) with both $h$ and $H$ included. Doing Feynman parametrization for the $d p$ integration one obtains

$$
\begin{aligned}
S_{\mu \nu}^{W} & =\frac{g_{\mu \nu}}{4} S^{W}, \\
S^{W} & =2 !\left(\frac{i}{16 \pi^{2}}\right) \int_{0}^{1} \frac{d x}{x(1-x)} \int_{0}^{(1-x)} d y \int_{0}^{(1-x-y)} d z J_{Q}^{W},
\end{aligned}
$$

plus terms suppressed by $1 / M_{H}^{2}$. This integral is finite. Note that the term $\sim 1 / R$ mentioned just above (A6) is not included. Here

$$
\begin{aligned}
J_{Q}^{W} \equiv & \int \frac{d r}{\left(r^{2}-M_{W}^{2}\right)^{2}\left(r^{2}-m_{t}^{2}\right)\left(r^{2}-Q\right)} \\
= & \frac{i}{16 \pi^{2}}\left[\frac{-1}{\left(m_{t}^{2}-M_{W}^{2}\right)\left(Q-M_{W}^{2}\right)}\right. \\
& \left.+\frac{m_{t}^{2}}{\left(m_{t}^{2}-M_{W}^{2}\right)^{2}}\left(\frac{\ln \left(Q / M_{W}^{2}\right)}{\left(Q-M_{W}^{2}\right)}-\frac{\ln \left(Q / m_{t}^{2}\right)}{\left(Q-m_{t}^{2}\right)}\right)\right],
\end{aligned}
$$

where

$$
\begin{aligned}
Q= & \frac{1}{x(1-x)}\left(m_{b}^{2}+x\left(m_{t}^{2}-m_{b}^{2}\right)+y\left(M_{h}^{2}-m_{b}^{2}\right)\right. \\
& \left.+z\left(M_{H}^{2}-m_{b}^{2}\right)\right) .
\end{aligned}
$$

Integrating over $z$ gives a suppression factor of order $1 / M_{H}^{2}$. Changing variables, one obtains an integral over $Q$ with $d z=x(1-x) d Q / M_{H}^{2}$,

$$
\int_{0}^{(1-x-y)} d z J_{Q}^{W}=\frac{i}{16 \pi^{2}} \frac{x(1-x)}{\left(M_{H}^{2}-m_{b}^{2}\right)}\left(f^{W}\left(Q_{1}\right)-f^{W}\left(Q_{0}\right)\right),
$$

where

$$
\begin{aligned}
f^{W}(Q) \equiv & \frac{1}{\left(m_{t}^{2}-M_{W}^{2}\right)}\left(-\ln \left(\frac{Q-M_{W}^{2}}{M_{W}^{2}}\right)\right. \\
& \left.+\frac{m_{t}^{2}}{\left(m_{t}^{2}-M_{W}^{2}\right)}\left(\operatorname{dilog}\left(\frac{Q}{m_{t}^{2}}\right)-\operatorname{dilog}\left(\frac{Q}{M_{W}^{2}}\right)\right)\right) .
\end{aligned}
$$

Here, the dilogarithmic function is in our case defined as

$$
\operatorname{dilog}(z)=\int_{1}^{z} d t \frac{\ln (t)}{(1-t)}=L i_{2}(1-z)
$$

Further,

$$
\begin{aligned}
Q_{1}= & \frac{1}{x(1-x)}\left(m_{b}^{2}+x\left(m_{t}^{2}-m_{b}^{2}\right)+y\left(M_{h}^{2}-m_{b}^{2}\right)\right. \\
& \left.+(1-x-y)\left(M_{H}^{2}-m_{b}^{2}\right)\right), \\
\text { and } \quad Q_{0}= & \frac{1}{x(1-x)}\left(m_{b}^{2}+x\left(m_{t}^{2}-m_{b}^{2}\right)+y\left(M_{h}^{2}-m_{b}^{2}\right)\right) \\
= & \frac{B}{x(1-x)},
\end{aligned}
$$

where $B$ is defined in (A4).

Now the quantity $S$ in (A7) may be split up as

$$
\begin{aligned}
S^{W} & =\left(\frac{i}{16 \pi^{2}}\right)^{2} 2 ! \int_{0}^{1} d x \int_{0}^{(1-x)} d y\left[f^{W}\left(Q_{1}\right)-f^{W}\left(Q_{0}\right)\right] \\
& =S_{1}^{W}-S_{0}^{W} .
\end{aligned}
$$

Here the quantity $S_{1}^{W}$ contains a term $\ln \left(M_{H}^{2}\right)$ corresponding to the divergent term $\ln \left(\Lambda^{2}\right)$ in [39] and (42). In order to find $S_{1}^{W}$ explicitly I use the asymptotic property for $Z \rightarrow \infty$

$$
\operatorname{dilog}(Z) \rightarrow-\frac{1}{2}(\ln (Z))^{2}
$$

Therefore, one obtains for $M_{H}^{2} \gg M_{h}^{2}$

$$
\begin{aligned}
& \left(-\operatorname{dilog}\left(\frac{Q_{1}}{M_{W}^{2}}\right)+\operatorname{dilog}\left(\frac{Q_{1}}{m_{t}^{2}}\right)\right) \\
& \rightarrow \ln \left(\frac{m_{t}^{2}}{M_{W}^{2}}\right) \cdot\left(\ln \left(\frac{M_{H}^{2}}{m_{t} M_{W}}\right)+\ln (\sigma)\right),
\end{aligned}
$$

where

$$
\sigma=\frac{(1-x-y)}{x(1-x)}
$$

Then one obtains

$$
\begin{aligned}
S_{1}^{W}= & \left(\frac{1}{16 \pi^{2}}\right)^{2} \frac{1}{M_{H}^{2}\left(m_{t}^{2}-M_{W}^{2}\right)}\left[-\left(\ln \left(\frac{M_{H}^{2}}{M_{W}^{2}}\right)+\frac{1}{2}\right)\right. \\
& \left.+\frac{m_{t}^{2} \ln \left(m_{t}^{2} / M_{W}^{2}\right)}{\left(m_{t}^{2}-M_{W}^{2}\right)}\left(\ln \left(\frac{M_{H}^{2}}{m_{t} M_{W}}\right)+\frac{1}{2}\right)\right],
\end{aligned}
$$

which may be manipulated into 


$$
S_{1}^{W}=\left(\frac{1}{16 \pi^{2}}\right)^{2} \frac{\left(\widetilde{C_{H}}\right)^{W} \cdot p_{W}\left(u_{t}\right)}{M_{H}^{2} M_{W}^{2}},
$$

where $\left(\widetilde{C_{H}}\right)^{W}$ is given in (49) and (50).

The term $S_{0}^{W}$ in (A14) contains the $\ln (R)$ term in (A5), and is given by

$$
S_{0}^{W}=\left(\frac{i}{16 \pi^{2}}\right)^{2} 2 ! \int_{0}^{1} d x \int_{0}^{(1-x)} d y f^{W}\left(Q_{0}\right)
$$

To see this clearly, instead of using (A5), one may use a trick by rewriting $I_{2}(R)$ in (A1)-(A3) as

$$
I_{2}(R)=2 \int_{R}^{\Lambda^{2}} d \rho \int \frac{d p}{\left(p^{2}-\rho\right)^{3}} .
$$

Also, one observes that $R=-x(1-x)\left(r^{2}-Q_{0}\right)$.
[1] M. Pospelov and A. Ritz, Ann. Phys. (Amsterdam) 318, 119 (2005).

[2] T. Fukuyama, Int. J. Mod. Phys. A 27, 1230015 (2012).

[3] W. Dekens, J. de Vries, J. Bsaisou, W. Bernreuther, C. Hanhart, and U.-G. Meißner, A. Nogga, and A. Wirzba, J. High Energy Phys. 07 (2014) 069.

[4] M. Jung and A. Pich, J. High Energy Phys. 04 (2014) 076.

[5] C. A. Baker, D. D. Doyle, P. Geltenbort, K. Green, M. G. D. van der Grinten, P. G. Harris, P. Iaydjiev, S. N. Ivanov et al., Phys. Rev. Lett. 97, 131801 (2006).

[6] E. P. Shabalin, Yad. Fiz. 31, 1665 (1980) [Sov. J. Nucl. Phys. 31, 864 (1980)].

[7] A. Czarnecki and B. Krause, Phys. Rev. Lett. 78, 4339 (1997).

[8] D. V. Nanopoulos, A. Yildiz, and P. H. Cox, Phys. Lett. B 87, 53 (1979).

[9] B. F. Morel, Nucl. Phys. B157, 23 (1979).

[10] M. B. Gavela, A. Le Yaouanc, L. Oliver, O. Pene, J. C. Raynal, and T. N. Pham, Phys. Lett. 109B, 215 (1982).

[11] I. B. Khriplovich and A. R. Zhitnitsky, Phys. Lett. 109B, 490 (1982).

[12] B. H. J. McKellar, S. R. Choudhury, X.-G. He, and S. Pakvasa, Phys. Lett. B 197, 556 (1987).

[13] J. O. Eeg and I. Picek, Phys. Lett. 130B, 308 (1983).

[14] J. O. Eeg and I. Picek, Nucl. Phys. B244, 77 (1984).

[15] T. Mannel and N. Uraltsev, Phys. Rev. D 85, 096002 (2012).

[16] W. Buchmuller and D. Wyler, Phys. Lett. 121B, 321 (1983).

[17] W. Altmannshofer, A. J. Buras, and P. Paradisi, Phys. Lett. B 688, 202 (2010).

[18] A. J. Buras, G. Isidori, and P. Paradisi, Phys. Lett. B 694, 402 (2011).

[19] J. Brod, U. Haisch, and J. Zupan, J. High Energy Phys. 11 (2013) 180.

[20] A. V. Manohar and M. B. Wise, Phys. Rev. D 74, 035009 (2006).

[21] G. Degrassi and P. Slavich, Phys. Rev. D 81, 075001 (2010).

[22] X.-G. He, C.-J. Lee, S.-F. Li, and J. Tandean, J. High Energy Phys. 08 (2014) 019.

[23] J. M. Arnold, B. Fornal, and M. B. Wise, Phys. Rev. D 87, 075004 (2013).

[24] A. Maiezza and M. Nemevšek, Phys. Rev. D 90, 095002 (2014).

[25] S. Bertolini, A. Maiezza, and F. Nesti, Phys. Rev. D 101, 035036 (2020).
[26] K. Fuyuto, J. Hisano, and E. Senaha, Phys. Lett. B 755, 491 (2016).

[27] W. Altmannshofer, R. Primulando, C.-T. Yu, and F. Yu, J. High Energy Phys. 04 (2012) 049.

[28] S. Fajfer and J. O. Eeg, Phys. Rev. D 89, 095030 (2014).

[29] A. Goudelis, O. Lebedev, and J-h. Park, Phys. Lett. B 707, 369 (2012).

[30] G. Blankenburg, J. Ellis, and G. Isidori, Phys. Lett. B 712, 386 (2012).

[31] R. Harnik, J. Kopp, and J. Zupan, J. High Energy Phys. 03 (2013) 026.

[32] A. Greljo, J. F. Kamenik, and J. Kopp, J. High Energy Phys. 07 (2014) 046.

[33] M. Gorbahn and U. Haisch, J. High Energy Phys. 06 (2014) 033.

[34] I. Doršner, S. Fajfer, A. Greljo, J. Kamenik, N. Košnik, and I. Nišandžic, J. High Energy Phys. 06 (2015) 108.

[35] S. M. Barr and A. Zee, Phys. Rev. Lett. 65, 21 (1990); 65, 2920(E) (1990).

[36] D.Chang, W. S. Hou, and W.-Y. Keung, Phys. Rev. D 48, 217 (1993).

[37] R. G. Leigh, S. Paban, and R. M. Xu, Nucl. Phys. B352, 45 (1991).

[38] J. Brod and D. Skodras, J. High Energy Phys. 01 (2019) 233.

[39] J. O. Eeg, Eur. Phys. J. C 78, 998 (2018).

[40] J. D. Bjorken and S. Weinberg, Phys. Rev. Lett. 38, 622 (1977).

[41] G. C. Branco, P. M. Ferreira, L. Lavoura, M. N. Rebelo, M. Sher, and J. P. Silva, Phys. Rep. 516, 1 (2012).

[42] W. Altmannshofer, J. Eby, S. Gori, M. Lotito, M. Martone, and D. Tuckler, Phys. Rev. D 94, 115032 (2016).

[43] F. J. Botella, G. C. Branco, M. N. Rebelo, and J. I. SilvaMarcos, Phys. Rev. D 94, 115031 (2016).

[44] B. Grzadkowski, O. M. Ogreid, and P. Osland, Phys. Rev. D 94, 115002 (2016).

[45] B. Grzadkowski, H. E. Haber, O. M. Ogreid, and P. Osland, J. High Energy Phys. 12 (2018) 056.

[46] J. M. Alves, F. J. Botella, G. C. Branco, F. Cornet-Gomez, M. Nebot, and J. P. Silva, Eur. Phys. J. C 78, 630 (2018).

[47] B. A. Ouazghour, A. Arhrib, R. Benbrik, M. Chabab, and L. Rahili, Phys. Rev. D 100, 035031 (2019). 
[48] W.-S. Hou, R. Jain, C. Kao, M. Kohda, B. McCoy, and A. Soni, Phys. Lett. B 795, 371 (2019).

[49] J. Herrero-Garcia, J. M. Nebot, F. Rajec, M. White, and A. G. Williams, J. High Energy Phys. 02 (2020) 147.

[50] I. de Medeiros Varzielas and J. Talbert, Phys. Lett. B 800, 135091 (2020).

[51] S. S. Correia, R. G. Felipe, and F. R. Joaquim, Phys. Rev. D 100, 115008 (2019).

[52] K. Fuyuto, W.-S. Hou, and E. Senaha, Phys. Rev. D 101, 011901 (2020).

[53] C.-R.Chen, Y.-X. Lin, C. S. Nugroho, R. Ramos, Y.-L. S. Tsai, and T.-C. Yuan, Phys. Rev. D 101, 035037 (2020).
[54] A. Crivellin, A. Kokulu, and C. Greub, Phys. Rev. D 87, 094031 (2013).

[55] M. Tanabashi et al., Phys. Rev. D 98, 030001 (2018).

[56] T. Bhattacharya, V. Cirigliano, R. Gupta, H-W. Lin, and B. Yoon, Phys. Rev. Lett. 115, 212002 (2015).

[57] T. Bhattacharya, V. Cirigliano, S. D. Cohen, R. Gupta, A. Joseph, H-W. Lin, and B. Yoon, Phys. Rev. D 92, 094511 (2015).

[58] N. Yamanaka, S. Hashimoto, T. Kaneko, and H. Ohki (JLQCD Collaboration), Phys. Rev. D 98, 054516 (2018).

[59] I. Doršner, S. Fajfer, A. Greljo, J. F. Kamenik, and N. Košnik, Phys. Rep. 641, 1 (2016). 